\title{
Moment Equations in Modeling a Stable Foreign Currency Exchange Market in Conditions of Uncertainty
}

\author{
Josef Diblík, ${ }^{1}$ Irada Dzhalladova, ${ }^{2}$ Mária Michalková, ${ }^{3}$ and Miroslava Růžičková ${ }^{3}$ \\ ${ }^{1}$ Department of Mathematics, Brno University of Technology, Brno 602 00, Czech Republic \\ ${ }^{2}$ Kyiv National Economic Vadym Hetman University, Kyiv 03680, Ukraine \\ ${ }^{3}$ The University of Žilina, Žilina 010 26, Slovakia \\ Correspondence should be addressed to Miroslava Růžičková; miroslava.ruzickova@fpv.uniza.sk
}

Received 26 September 2013; Accepted 12 November 2013

Academic Editor: Agacik Zafer

Copyright (C) 2013 Josef Diblík et al. This is an open access article distributed under the Creative Commons Attribution License, which permits unrestricted use, distribution, and reproduction in any medium, provided the original work is properly cited.

The paper develops a mathematical model of foreign currency exchange market in the form of a stochastic linear differential equation with coefficients depending on a semi-Markov process. The boundaries of the domain of its instability is determined by using moment equations.

\section{Introduction}

The economic growth of a given country is based on the government policy that includes numerous control moments. An important part of this policy is the correct financial policy, which defines the priorities in the development of financial relations and its function is to ensure the financial stability of the state. Finance and energy markets have been an active scientific field for some time, even though the development and applications of sophisticated quantitative methods in these areas are relatively new and referred to in a broader context as energy finance. Energy finance is often viewed as a branch of mathematical finance, yet this area continues to provide a rich source of issues that are fuelling new and exciting research developments [1].

The foreign currency exchange market is one of the most liquid financial markets with banks as major participants. Income from the foreign currency exchange transactions makes up a significant proportion of the banks income. The currency exchange risks associated with open positions are especially imminent in periods of significant fluctuations in exchange rates. The main feature of risky cases related to the market risk is that such cases occur as a result of adverse changes in the general market situation. Whenever such cases occur, the value of the assets has a tendency to decrease for a short-term period, causing liquidity gap.
In view of the disbalance of the foreign currency exchange market, the negative trade balance, the high inflation, an effective foreign currency exchange rate policy determining the optimal level of foreign currency exchange rates is an important problem.

Under such conditions, it is especially important to perceive the "bank" as a comprehensive dynamic system that works in the conditions of unstable economy under high foreign currency exchange risks. Thus, a more widespread use of economic-mathematical methods and models is necessary to study the processes taking place in the "bank", evaluating the effectiveness of its work and identifying the trends and ways to improve the management of the banking activities.

Significant scientific achievements in the field of banking and construction of some models can be found in [2, 3], and some economical models are studied in [4-6]. However, many other issues of bank practices require further research and elaboration of approaches to their solution. One aspect of the model is to build stable functioning of the foreign currency exchange transactions of the "bank" as a factor of effective functioning of the banking system in general $[7,8]$.

Most scientists understand under the category "financial stability of the banking system" the establishment of an effective mechanism preventing the emergence of banking crises and facilitating further development of economy. Depending 
on the tasks, the stability of the banks may be defined as in the model presented in this paper.

The paper develops a stability model of foreign currency bank transactions with semi-Markov fluctuations. An example illustrates the theory in the special case when the semiMarkov process can take three possible states. This means that a commercial bank operates in a foreign currency exchange market that can be in three states: stable foreign currency exchange market, market in the crisis, and market with currency restrictions. In the example, we assume that the bank remains in each state for the same period of time.

In addition, the present paper contains the necessary and sufficient conditions for the mean square stability and conditions for the $L_{2}$-stability of systems with semi-Markov coefficients and random transformations of solutions. There are constructed moment equations as a tool for studying the stochastic system stability which is working in uncertainty conditions.

\section{Statement of the Problem}

Let $(\Omega, \mathscr{F}, F, \mathbb{P})$ be a filtered probability space (or stochastic basis) consisting of a probability space $(\Omega, \mathscr{F}, \mathbb{P})$ and a filtration

$$
F=\left\{\mathscr{F}_{t}, \forall t \geq 0\right\} \subset \mathscr{F} .
$$

The space $\Omega$ is called the sample space, $\mathscr{F}$ is the set of all possible events (the $\sigma$-algebra), and $\mathbb{P}$ is some probability measure on $\Omega$. A family $\xi=\{\xi(t): t \geq 0\}$ of random variables $\xi(t): \Omega \rightarrow \mathbb{L}_{2}$ is called a continuous-time stochastic process on the state space $\mathbb{L}_{2}$. In our considerations, $\xi(t)$ is a random semi-Markov process and the state space $\mathbb{L}_{2}$ is the space of all random variables for which there exists squared mathematical expectation. On such a probability space, we consider initial problem formulated for the stochastic system of linear differential equations in the form

$$
\begin{gathered}
\frac{d x(t)}{d t}=A(\xi(t)) x(t), \\
x(0)=\varphi(\omega),
\end{gathered}
$$

where $A(\xi(t))$ is an $m \times m$ matrix whose elements depend on the semi-Markov process $\xi(t)$. The state function $x(t)$ is an $m$-dimensional column vector-function with the initial state $\varphi(\omega)$ at $t=0$. For simplicity, we denote

$$
A(\xi(t))=A_{k}, \quad \text { if } \xi(t)=\theta_{k}, k=1,2, \ldots, n,
$$

where $A_{k}$ are constant $m \times m$ matrices and $\theta_{k}$ are real numbers.

An $m$-dimensional vector-function $x(t)$ is called a solution of the initial value problem (2) and (3) if $x(t)$ satisfies (2) and initial condition (3) within the meaning of a strong solution of the initial Cauchy problem.

Our considerations are subject to the following assumptions.
Assumption 1. The random semi-Markov process $\xi(t)$ can take $n$ possible states

$$
\theta_{1}, \theta_{2}, \ldots, \theta_{n}
$$

with transition probabilities

$$
\begin{array}{r}
\pi_{s k}(t)=P\left\{\xi\left(t_{j+1}\right)=\theta_{s} \mid \xi\left(t_{j}\right)=\theta_{k}, j=1,2, \ldots\right\}, \\
s, k=1,2, \ldots, n,
\end{array}
$$

where $t_{0}=0<t_{1}<t_{2}<\cdots$ are the moments of time at which the jump from one state to another is realized.

If we fix any moment $t>0$, then the semi-Markov process takes some of states, $\xi(t)=\theta_{k}, k=1,2, \ldots, n$, and the state function $x(t) \equiv x(t, \omega)$ changes in accordance with the deterministic system of differential equations

$$
\frac{d x(t)}{d t}=A_{k} x(t), \quad k=1,2, \ldots, n .
$$

So the solution of such a system is in the form

$$
x(t)=e^{A_{k} t} \varphi(\omega), \quad k=1,2, \ldots, n .
$$

Assumption 2. The jumping time during which the process is in state $\theta_{s}$ before it jumps to state $\theta_{k}, s, k=1,2, \ldots, n$ is given by a discrete integer-valued random variable $T_{s k}$ whose probability density function is a known function $d_{s k}(t)$. Then, the intensity $q_{s k}(t)$ of the jump from state $\theta_{s}$ to state $\theta_{k}$ is given by the formula

$$
q_{s k}(t)=\pi_{s k}(t) d_{s k}(t), \quad s, k=1,2, \ldots, n,
$$

and the semi-Markov process $\xi(t)$ is defined by the transition intensity matrix

$$
Q(t)=\left(q_{s k}(t)\right)_{s, k=1}^{n},
$$

whose elements satisfy the relationships

$$
q_{s k}(t) \geq 0, \quad \int_{0}^{\infty} \sum_{s=1}^{n} q_{s k}(t) d t=\int_{0}^{\infty} q_{k}(t) d t=1,
$$

where $q_{k}(t)$ is the probability density of the elapsed time $T_{k}$ in state $\theta_{k}$ if the process jumps to it at time $t_{j}$. If $\psi_{k}(t)$ denotes the probability of the event that no jump takes place during the interval $\left(t_{j}, t_{j+1}\right)$, provided that the process jumps to the state $\theta_{k}$ at time $t_{j}$, then

$$
\psi_{k}(t)=\int_{t}^{\infty} q_{k}(\tau) d \tau, \quad k=1,2, \ldots, n .
$$

In our considerations, it will be convenient to denote the block-diagonal matrix,

$$
\Psi(t)=\operatorname{diag}\left(\psi_{1}(t), \psi_{2}(t), \ldots, \psi_{n}(t)\right) .
$$

Assumption 3. At the moments of jumps $t_{j}, j=1,2, \ldots$ that are caused by some perturbations, solutions of (2) submit to the random transformations

$$
x\left(t_{j}+0\right)=C_{s k} x\left(t_{j}-0\right), \quad s, k=1, \ldots, n,
$$

where $C_{s k}$ are $m \times m$ constant matrices and $\operatorname{det} C_{s k} \neq 0$. 
Our aim is to transform the stochastic system with random coefficients to a deterministic system with solutions whose stability can be considered by using classical methods. To complete this task below, we present a method of moment equations. We will show that the method is effective and useful for solving an economical model problem.

\section{Construction of the Moment Equations}

We define the moments of the first or second order of a random variable $x$ before we derive the moments equations. We use some notation. In the sequel, $\mathbb{E}_{m}$ denotes an $m$-dimensional Euclidean space, functions $f_{k}(t, x), k=$ $1,2, \ldots, n$ are the particular density functions of the random variable $x$, and the vector-function

$$
f(t, x)=\left(f_{1}(t, x), f_{2}(t, x), \ldots, f_{n}(t, x)\right)^{T},
$$

where the operation $T$ denotes transposition, is called the vector of particular density functions. Moreover, we define

$$
\begin{gathered}
S(t):=\left(q_{s k} S_{s k}\right)_{s, k=1}^{n}, \quad s, k=1, \ldots, n, \\
R(t):=\operatorname{diag}\left(R_{1}(t), \ldots, R_{n}(t)\right),
\end{gathered}
$$

where $S_{s k}$ are operators defined, for a given function $f$, as

$$
S_{s k} f(t, x) \equiv f_{k}\left(t, C_{s k}^{-1} x\right) \operatorname{det} C_{s k}^{-1}
$$

and $R_{k}, k=1, \ldots, n$ are operators defined, for a given function $f$, as

$$
R_{k}(t) f(t, x) \equiv f_{k}\left(t, e^{-A_{k} t} x\right) \operatorname{det}\left(e^{-A_{k} t}\right) .
$$

Definition 4. Let $x \in \mathbb{E}_{m}$ be a continuous random variable depending on a random semi-Markov process $\xi(t)$ with $n$ possible states $\theta_{k}, k=1,2, \ldots, n$. The $n$-dimensional column vectors $E^{(1)}\{x(t)\}$ and $n \times n$ matrices $E^{(2)}\{x(t)\}$ of the form

$$
E^{(1)}\{x(t)\}=\sum_{k=1}^{n} E_{k}^{(1)}\{x(t)\}, \quad E^{(2)}\{x(t)\}=\sum_{k=1}^{n} E_{k}^{(2)}\{x(t)\},
$$

where

$$
\begin{aligned}
E_{k}^{(1)}\{x(t)\} & =\int_{\mathbb{E}_{m}} x f_{k}(t, x) d x, \\
E_{k}^{(2)}\{x(t)\} & =\int_{\mathbb{E}_{m}} x x^{T} f_{k}(t, x) d x,
\end{aligned}
$$

are called moments of the first or second order of the random variable $x$, respectively. The values $E_{k}^{(1)}\{x(t)\}$ and $E_{k}^{(2)}\{x(t)\}$, $k=1, \ldots, n$ are called particular moments of the first or second order, respectively.

Theorem 5. Let the coefficients of the linear differential system (2) depend on a random semi-Markov process $\xi(t)$ with transition intensity matrix (10) and, for solutions of system (2), there occur jumps (14) simultaneously with jumps of the process $\xi(t)$. Then, the following three statements are true.
(1) The stochastic process $(x(t), \xi(t))$ is defined by the operator equation

$$
f(t, x)=L(t) f(0, x),
$$

where the matrix operator $L(t) \equiv\left(L_{i j}(t)\right)_{i, j=1}^{n}$ satisfies

$$
L(t)=\psi(t) R(t)+\int_{0}^{t} L(t-\tau) S(\tau) R(\tau) d \tau .
$$

(2) The vectors of particular moments of first order satisfy

$$
\begin{aligned}
E_{k}^{(1)}\{x(t)\}= & \psi_{k}(t) e^{A_{k} t} E_{k}^{(1)}\{x(0)\} \\
& +\int_{0}^{t} \psi(t-\tau) e^{A_{k}(t-\tau)} Z_{k}(\tau) d \tau,
\end{aligned}
$$

where

$$
\begin{aligned}
Z_{k}(t)= & \sum_{s=1}^{n} q_{k s}(t) C_{k s} e^{A_{s} t} E_{s}^{(1)}\{x(0)\} \\
& +\int_{0}^{t} \sum_{s=1}^{n} q_{k s}(t-\tau) C_{k s} e^{A_{s}(t-\tau)} Z_{s}(\tau) d \tau
\end{aligned}
$$

and $k=1, \ldots, n$.

(3) The matrix of particular moments of second order satisfies

$$
\begin{aligned}
E_{k}^{(2)}\{x(t)\}= & \psi_{k}(t) e^{A_{k} t} E_{k}^{(2)}\{x(0)\} e^{A_{k}^{T} t} \\
& +\int_{0}^{t} \psi_{k}(t-\tau) e^{A_{k}(t-\tau)} W_{k}(\tau) e^{A_{k}^{T}(t-\tau)} d \tau,
\end{aligned}
$$

where

$$
\begin{aligned}
W_{k}(t)= & \sum_{s=1}^{n} q_{k s}(t) C_{k s} e^{A_{s} t} E_{s}^{(2)}\{x(0)\} e^{A_{s}^{T} t} C_{k s}^{T} \\
& +\int_{0}^{t} \sum_{s=1}^{n} q_{k s}(t-\tau) C_{k s} e^{A_{s}(t-\tau)} W_{s}(\tau) e^{A_{s}^{T}(t-\tau)} C_{k s}^{T} d \tau,
\end{aligned}
$$

and $k=1, \ldots, n$.

Proof. (1) The stochastic process $(x(t), \xi(t))$ is also semiMarkov because all probabilistic properties of the process for $t>t_{j}$ are defined by particular probability density functions at the moment $t_{j}$ of jump. Thus, there exists a linear operator $L(\tau)$ such that

$$
f\left(t_{j}+\tau, x\right)=L(\tau) f\left(t_{j}, x\right), \quad j=0,1,2, \ldots
$$

Let the stochastic process $\xi(t)$ move to state $\theta_{k}$ at the moment $t=0$. Then,

$$
\begin{array}{r}
p_{k}(0)=1, \quad p_{s}(0)=0, \quad \text { for } s \neq k, \quad s=1, \ldots, n, \\
f_{k}(0, x) \geq 0, \quad \int_{E_{m}} f_{k}(0, x) d x=1, \quad f_{s}(0, x) \equiv 0, \\
s \neq k .
\end{array}
$$


For particular density functions, when $t \geq 0$, we obtain

$$
f_{s}(t, x)=L_{s k}(t) f_{k}(0, x), \quad k, s=1, \ldots, n .
$$

Also, with probability $\psi_{k}(t)$, in view of $x(0)=e^{-A_{k} t} x$, we obtain the equality

$$
\begin{array}{r}
f_{k}(t, x)=f_{k}\left(0, e^{-A_{k} t} x\right) \operatorname{det}\left(e^{-A_{k} t}\right), \quad f_{s}(t, x) \equiv 0, \\
k \neq s .
\end{array}
$$

On the interval $(\tau, \tau+d \tau)$, there could be a jump of the stochastic process $\xi(\tau)$ from state $\theta_{k}$ to state $\theta_{s}$ with probability $q_{k s}(\tau) d \tau$. Taking into account that functions $q_{k s}(t)$ are continuous, we obtain the probability

$$
P\left\{\tau<t_{1}<\tau+d \tau\right\}=\int_{\tau}^{\tau+d \tau} q_{k}(s) d s \approx q_{k}(\tau) d \tau .
$$

After a jump at the moment lying between moments $\tau$ and $\tau+d \tau$, we can use (27). At the moment $t_{1}$ of the first jump of the stochastic process $\xi(t)$, in accordance with (14), we have

$$
f_{s}\left(t_{1}+0, x\right)=f_{k}\left(t_{1}-0, C_{s k}^{-1} x\right) \operatorname{det} C_{s k}^{-1} .
$$

Therefore, by using operators $R, S$ defined in (16), to remain in state $\theta_{k}$ at the moment $t$, we get

$$
\begin{aligned}
& L_{k k}(t) f_{k}(0, x) \\
& =\psi_{k}(t) R_{k}(t) f_{k}(0, x) \\
& \quad+\int_{0}^{t} \sum_{s=1}^{n} L_{k s}(t-\tau) q_{s k}(\tau) S_{s k} R_{k}(\tau) f_{k}(0, x) d \tau, \\
& \quad k=1, \ldots, n .
\end{aligned}
$$

For transition from state $\theta_{k}$ to state $\theta_{\tau}$ at the moment $t$, we obtain

$$
\begin{aligned}
L_{\tau k}(t) & f_{k}(0, x) \\
& =\int_{0}^{t} \sum_{s=1}^{n} L_{\tau s}(t-\tau) q_{s k}(\tau) S_{s k} R_{k}(\tau) f_{k}(0, x) d \tau,
\end{aligned}
$$

where $\tau \neq k, \tau, k=1, \ldots, n$.

These two systems can be written in the form (22).

(2) Before we derive the system of moment equations in (23), we establish an auxiliary operator equation. Let us find the solution of (22) in the form

$$
L(t)=\psi(t) R(t)+\int_{0}^{t} \psi(t-\tau) R(t-\tau) U(\tau) d \tau,
$$

where $U$ is an unknown matrix. If we put $L(t)$ expressed in the form (36) into (22), we obtain

$$
\begin{aligned}
\int_{0}^{t} \psi(t-\tau) R(t-\tau) U(\tau) d \tau \\
\quad=\int_{0}^{t} \psi(t-\tau) T(t-\tau) S(\tau) R(\tau) d \tau \\
\quad+\int_{0}^{t} d \tau \int_{0}^{t-\tau} \psi(t-\tau-s) R(t-\tau-s) U(s) S(\tau) R(\tau) d s .
\end{aligned}
$$

After substituting $r=\tau+s, \tau=\tau$ in the double integral and after changing the order of integration, we obtain

$$
\begin{gathered}
\int_{0}^{t} d \tau \int_{0}^{t-\tau} \psi(t-\tau-s) R(t-\tau-s) U(s) S(\tau) R(\tau) d s \\
\quad=\int_{0}^{t} \psi(t-r)\left(\int_{0}^{r} U(r-\tau) S(\tau) R(\tau) d \tau\right) d r .
\end{gathered}
$$

Therefore, a suitable matrix $U$ in (22) is the matrix

$$
U(t)=S(t) R(t)+\int_{0}^{t} U(t-\tau) S(\tau) R(\tau) d \tau .
$$

In the other way, we can find a matrix $U(t)$ as a solution of (36) in the form

$$
U(t)=S(t) R(t)+\int_{0}^{t} S(t-\tau) R(t-\tau) V(\tau) d \tau,
$$

where $V$ is an unknown matrix. From this, we get

$$
V(t)=S(t) R(t)+\int_{0}^{t} V(t-\tau) S(\tau) R(\tau) d \tau .
$$

A comparison of (39) and (41) implies that we can set $V(t) \equiv$ $U(t)$. Then, (40) can be written as

$$
U(t)=S(t) R(t)+\int_{0}^{t} S(t-\tau) R(t-\tau) U(\tau) d \tau,
$$

or

$$
U(t)=S(t) R(t)+\int_{0}^{t} S(\tau) R(\tau) U(t-\tau) d \tau
$$

Multiplying (36) and (38) on the right by the vector $f(0, x)$, we obtain

$$
\begin{aligned}
f(t, x)= & \psi(t) R(t) f(0, x) \\
& +\int_{0}^{t} \psi(t-\tau) R(t-\tau) U(\tau) f(0, x) d \tau, \\
U(t) f(0, x)= & S(t) R(t) f(0, x) \\
& +\int_{0}^{t} S(t-\tau) R(t-\tau) U(\tau) f(0, x) d \tau .
\end{aligned}
$$

Denote

$$
\begin{gathered}
h(t, x)=U(t) f(0, x), \\
h(t, x)=\left(h_{1}(t, x), h_{2}(t, x), \ldots, h_{n}(t, x)\right)^{T},
\end{gathered}
$$

and

$$
\begin{gathered}
z_{k}(t)=\int_{\mathbb{E}_{m}} x h_{k}(t, x) d x, \\
W_{k}(t)=\int_{\mathbb{E}_{m}} x x^{T} h_{k}(t, x) d x,
\end{gathered}
$$


where $k=1, \ldots, n$. The system (44) can be rewritten into the scalar form

$$
\begin{aligned}
f_{k}(t, x)= & \psi_{k}(t) R_{k}(t) f_{k}(0, x) \\
& +\int_{0}^{t} \psi_{k}(t-\tau) R_{k}(t-\tau) h_{k}(\tau, x) d \tau, \\
h_{k}(t, x)= & \sum_{s=1}^{n} q_{k s}(t) S_{k s} R_{s}(t) f_{s}(0, x) \\
& +\int_{0}^{t} \sum_{s=1}^{n} q_{k s}(t-\tau) S_{k s} R_{s}(t-\tau) h_{s}(\tau, x) d \tau, \\
& k=1, \ldots, n .
\end{aligned}
$$

The system of (23) can be obtained by multiplying each equation of (48) by vector $x$ and integrating it over the space $\mathbb{E}_{m}$. In doing so, it is necessary to use

$$
\begin{aligned}
& \int_{\mathbb{E}_{m}} x f_{k}(t, x) d x=E_{k}^{(1)}\{x(t)\} \\
& \int_{\mathbb{E}_{m}} x R_{k}(t) f_{k}(0, x) \\
& =\int_{\mathbb{E}_{m}} x f_{k}\left(0, e^{-A_{k} t} x\right) \operatorname{det} e^{-A_{k} t} d x \\
& =\int_{\mathbb{E}_{m}} e^{A_{k} t} y f_{k}(0, y) d y=e^{A_{k} t} E_{k}^{(1)}\{x(t)\}, \\
& \int_{\mathbb{E}_{m}} x R_{k}(t-\tau) h_{k}(\tau, x) d x \\
& =\int_{\mathbb{E}_{m}} x h_{k}\left(\tau, e^{-A_{k}(t-\tau)}\right) \operatorname{det} e^{-A_{k}(t-\tau)} d x \\
& =e^{A_{k}(t-\tau)} \int_{\mathbb{E}_{m}} y h_{k}(\tau, y) d y=e^{A_{k}(t-\tau)} z_{k}(\tau), \\
& \int_{\mathbb{E}_{m}} x S_{k s} R_{k}(t) f_{s}(0, x) d x \\
& =\int_{\mathbb{E}_{m}} x S_{k s} f_{s}\left(0, e^{-A_{s} t} x\right) \operatorname{det} e^{-A_{s} t} d x \\
& =\int_{\mathbb{E}_{m}} x f_{s}\left(0, e^{-A_{s} t} C_{k s}^{-1} x\right) \operatorname{det} e^{-A_{s} t} C_{k s}^{-1} d x=C_{k s} e^{A_{s} t}, \\
& \int_{\mathbb{E}_{m}} x f_{s}(0, x) d x=C_{k s} e^{A_{s} t} E_{s}^{(1)}\{x(0)\} .
\end{aligned}
$$

(3) The system of (25) can be obtained by multiplying each equation in (48) by matrix $x x^{T}$ and integrating it over the space $\mathbb{E}_{m}$ by using matrix equalities

$$
\begin{aligned}
\int_{\mathbb{E}_{m}} x x^{T} R_{k}(t) f_{k}(0, x) d x \\
=\int_{\mathbb{E}_{m}} x x^{T} f_{k}\left(0, e^{-A_{k} t} x\right) \operatorname{det} e^{-A_{k} t} d x
\end{aligned}
$$

$$
\begin{aligned}
&=\int_{\mathbb{E}_{m}} e^{A_{k} t} y y^{T} e^{A_{k}^{T} t} f_{k}(0, y) d y=e^{A_{k} t} E_{k}^{(2)}\{x(0)\} e^{A_{k}^{T} t}, \\
& \int_{\mathbb{E}_{m}} x x^{T} S_{k s} R_{s}(t) f_{s}(0, x) d x \\
&=\int_{\mathbb{E}_{m}} x x^{T} f_{s}\left(0, e^{-A_{k} t} C_{k s}^{-1} x\right) \operatorname{det} e^{-A_{k} t} \operatorname{det} C_{k s}^{-1} d x \\
&=C_{k s} e^{A_{s} t} E_{s}^{(2)}\{x(0)\} e^{A_{s}^{T} t} C_{k s}^{T} .
\end{aligned}
$$

\section{Necessary and Sufficient Conditions of $L_{2}$-Stability}

Several different stability definitions are useful. Here, we recall the mean stability and the mean square stability definitions, the $L_{2}$-stability, and the classical definition of asymptotic stability.

Definition 6. The trivial solution of system (2) is said to be mean square stable on the interval $[0, \infty)$ if, for each $\varepsilon>0$, there exists $\delta>0$ such that any solution $x(t)$ corresponding to the initial data $x(0)$ exists for all $t \geq 0$ and the mathematical expectation

$$
E^{(1)}\left\{\|x(t)\|^{2}\right\}<\varepsilon, \text { whenever } t \geq 0,\|x(0)\|<\delta .
$$

The mean stability of the zero solution of system (2) is much defined in the same way with only $\|x(t)\|^{2}$ being replaced by $\|x(t)\|$.

Definition 7. The trivial solution of system (2) is said to be asymptotically mean square stable on the interval $[0, \infty)$ if it is stable and, moreover,

$$
\lim _{t \rightarrow \infty} E^{(2)}\{x(t)\}=0 .
$$

Remark 8. It is obvious that the mean stability of the zero solution of system (2) is equivalent to the asymptotic stability of the solutions of system (23) and (24) and the mean square stability of the solutions of system (2) is equivalent to the asymptotic stability of the solutions of system (25) and (26).

Definition 9. The trivial solution of the differential systems (2) is said to be $L_{2}$-stable if the integral

$$
\int_{0}^{\infty} E^{(1)}\left\{\|x(t)\|^{2}\right\} d t
$$

converges.

Remark 10. It is easy to see that the integral (53) converges if and only if the matrix integral

$$
\int_{0}^{\infty} E^{(2)}\{x(t)\} d t
$$

is convergent. 
Lemma 11. The following three inequalities hold:

(1) $E_{k}^{(2)}\{x(t)\} \geq 0, k=1, \ldots, n$.

(2) $e^{A_{k} t} E_{k}^{(2)}\{x(0)\} e^{A_{k}^{T} t} x \geq 0, k=1, \ldots, n$.

(3) $C_{k s} e^{A_{s} t} E_{s}^{(2)}\{x(0)\} e^{A_{s}^{T} t} C_{k s}^{T} \geq 0, k, s=1, \ldots, n$.

Proof. All inequalities follow from property $f_{k}(t, x) \geq 0, k=$ $1, \ldots, n$ in accordance with (23) and (24).

It is convenient to derive some sufficient and necessary conditions of $L_{2}$-stability by using a matrix operator with suitable properties. Such an operator is defined by the following lemma.

Lemma 12. The linear matrix operators $N_{k s}(t), k, s=1, \ldots, n$, defined as

$$
\begin{aligned}
N_{k s}(t) & \circ W_{s}(t) \\
& :=\int_{0}^{t} q_{k s}(t-\tau) C_{k s} e^{A_{s}(t-\tau)} W_{s}(\tau) e^{A_{s}^{T}(t-\tau)} C_{k s}^{T} d \tau,
\end{aligned}
$$

are monotonous.

Proof. Because $q_{k s}(t-\tau) \geq 0$, in accordance with the third statement of Lemma 11, we have

$$
x^{T} N_{k s}(t) \circ W_{s}(t) x \geq 0, \text { for } x \geq 0 .
$$

So $W_{s}(\tau) \geq 0$ implies $N_{k s}(t) \circ W_{s}(t) \geq 0$ and the operator is monotone.

Remark 13. The linear monotonous operator $N_{k s}(t)$ transforms any symmetric matrix $D$ into a symmetric matrix $N_{k s}(t) \circ D$. Its monotonicity guarantees that inequality $D_{1} \geq$ $D_{2}$ implies inequality

$$
N_{k s}(t) \circ D_{1} \geq N_{k s}(t) \circ D_{2} .
$$

Lemma 14. The symmetric matrices $W_{k}(t), k=1, \ldots, n$ defined by (26) satisfy the inequalities $W_{k}(t) \geq 0, k=1, \ldots, n$.

Proof. System (26) can be rewritten in the form

$$
\begin{aligned}
W_{k}(t)= & \sum_{s=1}^{n} q_{k s}(t) C_{k s} e^{A_{s} t} E_{s}^{(2)}\{x(0)\} e^{A_{s}^{T} t} C_{k s}^{T} \\
& +\sum_{s=1}^{n} N_{k s}(t) \circ W_{s}(t),
\end{aligned}
$$

$k=1, \ldots, n$, by using the matrix operators (55). System (26) as well as system (58) can be solved by the method of successive approximations

$$
\begin{array}{r}
W_{k}^{(0)}(t)=\sum_{s=1}^{n} q_{k s}(t) C_{k s} e^{A_{s} t} E_{s}^{(2)}\{x(0)\} e^{A_{s}^{T} t} C_{k s}^{T}, \\
W_{k}^{(l+1)}(t)=W_{k}^{(0)}(t)+\sum_{s=1}^{n} N_{k s}(t) \circ W_{s}^{(l)}(t), \\
l=1,2, \ldots
\end{array}
$$

Hence, $W_{k}^{(0)}(t) \geq 0$ and $W_{k}^{(l)}(t) \geq 0$ implies $W_{k}^{(l+1)} \geq 0, k=$ $1, \ldots, n$. So the solution of system (58),

$$
W_{k}(t)=\lim _{l \rightarrow \infty} W_{k}^{(l)}(t)
$$

satisfies $W_{k}(t) \geq 0, k=1, \ldots, n$.

Now, we rewrite the moment equations (25) and (26) into a more compact form by using the denotations

$$
\begin{array}{r}
D_{k}=\int_{0}^{\infty} E_{k}^{(2)}\{x(t)\} d t, \quad W_{k}=\int_{0}^{\infty} W_{k}(\tau) d \tau, \\
k=1, \ldots, n .
\end{array}
$$

Then, integrating systems (25) and (26) from 0 to $\infty$ with respect to $t$, we obtain

$$
\begin{array}{r}
D_{k}=\int_{0}^{\infty} \psi_{k}(t) e^{A_{k} t}\left(E_{k}^{(2)}\{x(0)\}+W_{k}\right) e^{A_{k}^{T} t} d t, \\
k=1, \ldots, n,
\end{array}
$$

$$
\begin{array}{r}
W_{k}=\sum_{s=1}^{n} \int_{0}^{\infty} q_{k s}(t) C_{k s} e^{A_{s} t}\left(E_{s}^{(2)}\{x(0)\}+W_{s}\right) e^{A_{s}^{T} t} C_{k s}^{T} d t, \\
k=1, \ldots, n .
\end{array}
$$

Corollary 15. Let the zero solution of the system (2) with jumps (14) at random time moments $t_{j}, j=0,1,2, \ldots$ determined by jumps of stochastic process $\xi(t)$ be $L_{2}$-stable. Then, the integrals

$$
I_{k}=\int_{0}^{\infty} \psi_{k}(t) e^{A_{k} t} E_{k}^{(2)}\{x(0)\} e^{A_{k}^{T} t} d t, \quad k=1, \ldots, n
$$

are convergent.

Proof. This immediately follows from Lemma 14. In fact, since $W_{k} \geq 0$, then $D_{k} \geq I_{k}, k=1, \ldots, n$.

Theorem 16. Let the integrals (64) be convergent. Then, the zero solution of system (2) is $L_{2}$-stable if and only if the solutions $W_{k} \geq 0, k=1, \ldots, n$ of system (63) are bounded.

Proof. (1) Sufficiency. Integrating system (59) from 0 to $\infty$ with respect to $t$, using notation $W_{k}^{(l)}=\int_{0}^{\infty} W_{k}^{(l)}(t) d t$, $k=1, \ldots, n$, we obtain the system of matrix successive approximations

$$
\begin{array}{r}
W_{k}^{(l+1)}=W_{k}^{(0)}+\sum_{s=1}^{n} N_{k s} \circ W_{s}^{(l)}, \quad k=1, \ldots, n, \\
l=0,1,2, \ldots
\end{array}
$$

As the linear operators $N_{k s}, k, s=1, \ldots, n$ defined by (55) are monotonous, the zero solution of system (2) is $L_{2}$-stable if the sequence of matrices $W_{k}^{(l)}, l=0,1,2, \ldots$ is convergent. 
(2) Necessity. Let us assume that the solution $W_{k}=Z_{k} \geq 0$, $k=1, \ldots, n$ of the system

$$
W_{k}=W_{k}^{(0)}+\sum_{s=1}^{n} N_{k s} \circ W_{s}, \quad k=1, \ldots, n
$$

is bounded. Obviously, $Z_{k} \geq W_{k}^{(0)}, k=1, \ldots, n$ and, in view of successive approximations (65), we get

$$
\begin{aligned}
W_{k}^{(l+1)} & =W_{k}^{(0)}+N_{k s} \circ W_{s}^{(l)} \\
& \leq W_{k}^{(0)}+N_{k s} \circ Z_{s}=Z_{k}, \quad k=1, \ldots, n .
\end{aligned}
$$

So, for all $l=0,1,2, \ldots$, we have $W_{k}^{(l)} \leq Z_{k}, k=1, \ldots, n$.

Next, from (65), we obtain

$$
\begin{aligned}
W_{k}^{(l+1)}= & W_{k}^{(0)}+N_{k s} \circ W_{s}^{(l)} \geq W_{k}^{(0)} \\
& +N_{k s} \circ W_{s}^{(l-1)}=W_{k}^{(l)}, \quad k=1, \ldots, n .
\end{aligned}
$$

Moreover, because $W_{k}^{(0)} \geq 0, W_{k}^{(l+1)} \geq W_{k}^{(l)}, k=1, \ldots, n$ is satisfied for all $l=0,1, \ldots$.

Finally, the boundedness and monotonicity of the matrix sequences $W_{k}^{(l)}, l=0,1, \ldots$ imply the existence of limits

$$
W_{k}=\lim _{l \rightarrow \infty} W_{k}^{(l)}
$$

$k=1, \ldots, n$. Consequently, independently of the initial value $W_{k}^{(0)}$, in view of $0 \leq W_{k}^{(l)} \leq Z_{k}$, the linear operator $N=$ $\left(N_{k s}\right)_{k, s=1}^{n}$ has the spectral radius less than 1 . This means that system (65) has a unique solution $W_{k}=Z_{k}, k=1, \ldots, n$.

\section{Model Problem}

The functioning of the foreign currency exchange market in conditions of uncertainty can be modelled by using stochastic differential equations. Such convenient mathematical model is the scalar case of the initial problem (2), (3), that is, the initial problem formulated for the stochastic linear differential equation

$$
\begin{gathered}
\frac{d x(t)}{d t}=a(\xi(t)) x(t), \\
x(0)=\varphi(\omega),
\end{gathered}
$$

where coefficient $a$ depends on a semi-Markov process $\xi(t)$. The possible states $\theta_{1}, \ldots, \theta_{n}$ of the stochastic process $\xi(t)$ express the conditions in which the bank works, for example, in a currency crisis, in a stable foreign currency exchange market, and so forth. Let the stochastic process $\xi(t)$ take the states $\theta_{k}, k=1,2, \ldots, n$. If $\xi(t)=\theta_{k}$, we denote $a(\xi(t))=a_{k}$. Further assume that the intensities $q_{s k}(t)$ are determined by the formulas

$$
\begin{aligned}
& q_{s s}(t)=0, \\
& q_{s k}(t)= \begin{cases}\frac{1}{T_{s k}} & \text { for } 0 \leq t<T_{s k}, \\
0 & \text { for } t \geq T_{s k},\end{cases}
\end{aligned}
$$

where $s, k=1,2, \ldots, n$.
Perturbations in the foreign currency exchange market cause the changes of the stochastic process $\xi(t)$, and consequently, solutions of (2) in this scalar case are subject to the random transformations

$$
x\left(t_{j}+0\right)=p_{k} x\left(t_{j}-0\right), \quad p_{k} \neq 0, k=1, \ldots, n
$$

at the moments of jumps $t_{j}, j=1,2, \ldots$.

We derive the domain of stability of the foreign currency exchange market using the results of the previous section. The moment equations in (25) for $E_{k}^{(2)}\{x(t)\}, k=1, \ldots, n$ take, in the scalar case, the form

$$
\begin{aligned}
& E_{k}^{(2)}\{x(t)\}= \psi_{k} e^{2 a_{k} t} E_{k}^{(2)}\{x(0)\} \\
&+\int_{0}^{t} \psi_{k}(t-\tau) e^{2 a_{k}(t-\tau)} W_{k}(\tau) d \tau, \\
& W_{k}(t)= \sum_{s=1}^{n} q_{k s}(t) C_{k s}^{2} e^{2 a_{s} t} E_{s}^{(2)}\{x(0)\} \\
&+\int_{0}^{t} \sum_{s=1}^{n} q_{k s}(t-\tau) C_{k s}^{2} e^{2 a_{s}(t-\tau)} W_{s}(\tau) d \tau, \\
& \quad k=1, \ldots, n .
\end{aligned}
$$

By definition, the zero solution of (75) is asymptotically stable if $E^{(2)}\{x(t)\} \rightarrow 0$ for $t \rightarrow \infty$.

Lemma 17. If

$$
\lim _{t \rightarrow \infty} E_{k}^{(2)}\{x(t)\}=0, \quad k=1, \ldots, n,
$$

then

$$
\lim _{t \rightarrow \infty} \psi_{k}(t) e^{2 a_{k} t}=0, \quad k=1, \ldots, n
$$

Proof. Formula (20) implies $E_{k}^{(2)}\{x(t)\} \geq 0, k=1, \ldots, n$ if $t \geq 0$. Therefore, $W_{k}(t) \geq 0, k=1, \ldots, n, t \geq 0$. From (75), it follows that inequalities

$$
E_{k}^{(2)}\{x(t)\} \geq \psi_{k} e^{2 a_{k} t} E_{k}^{(2)}\{x(0)\}, \quad k=1, \ldots, n
$$

are always satisfied. Then, for any constant $E_{k}^{(2)}\{x(0)\}$, property (77) implies (78).

Note that, in general, condition (78) does not imply the property (77). In the following theorem, it is shown what additional assumptions are needed.

Theorem 18. Let (78) hold and let

$$
\int_{0}^{\infty} \psi_{k}(t) e^{2 a_{k} t} d t<\infty, \quad k=1, \ldots, n
$$

Then, if there exist limits

$$
\lim _{t \rightarrow \infty} W_{k}(t)=0, \quad k=1, \ldots, n
$$

limits (77) exist too. 
Proof. We denote

$$
I_{k}=\int_{0}^{\infty} \psi_{k}(t) e^{2 a_{s} t} d t, \quad I_{k} \geq 0, k=1, \ldots, n .
$$

As the integrals $I_{k}, k=1, \ldots, n$ are convergent, for all $\varepsilon>0$, there exists $T_{1}>0$ such that, for all $t>T_{1}$, the inequalities

$$
\int_{t}^{\infty} \psi_{k}(t) e^{2 a_{k} t}<\varepsilon, \quad k=1, \ldots, n
$$

hold. Similarly, assumption (81) means that $\forall \mathcal{E}>0 \exists T_{2}>0$ such that $\forall t>T_{2}$ the inequalities

$$
0 \leq W_{k}(t)<\varepsilon, \quad k=1, \ldots, n
$$

hold. Moreover, there exists constant $W_{0}$ such that

$$
\left|W_{k}(t)\right| \leq W_{0}, \quad k=1, \ldots, n, t \geq T_{2} .
$$

The integral part of (75) can be now estimated if $t>T_{1}+T_{2}$. We have

$$
\begin{aligned}
\int_{0}^{t} \psi_{k}( & t-\tau) e^{2 a_{k}(t-\tau)} W_{k}(\tau) d \tau \\
= & \int_{0}^{T_{1}+T_{2}} \psi_{k}(t) e^{2 a_{k} \tau} W_{k}(t-\tau) d \tau \\
& +\int_{T_{1}+T_{2}}^{t} \psi_{k}(t) e^{2 a_{k} \tau} W_{k}(t-\tau) d \tau \\
\leq & I_{k} \varepsilon+W_{0} \varepsilon .
\end{aligned}
$$

$$
\Delta(p)=\left|\begin{array}{cccc}
1-\Theta_{11}\left(p-2 a_{1}\right) C_{11}^{2} & -\Theta_{12}\left(p-2 a_{2}\right) C_{12}^{2} & \cdots & -\Theta_{1 n}\left(p-2 a_{n}\right) C_{1 n}^{2} \\
-\Theta_{21}\left(p-2 a_{1}\right) C_{21}^{2} & 1-\Theta_{22}\left(p-2 a_{2}\right) C_{22}^{2} & \cdots & -\Theta_{2 n}\left(p-2 a_{n}\right) C_{2 n}^{2} \\
\vdots & \vdots & \ddots & \vdots \\
-\Theta_{n 1}\left(p-2 a_{1}\right) C_{n 1}^{2} & -\Theta_{n 2}\left(p-2 a_{2}\right) C_{n 2}^{2} & \cdots & 1-\Theta_{n n}\left(p-2 a_{n}\right) C_{n n}^{2}
\end{array}\right| .
$$

The singular points of mappings $\Theta_{k s}(p), k, s=1, \ldots, n$ are determined by the roots of $\Delta(p)=0$. If all the functions $\Theta_{k s}(p), k, s=1, \ldots, n$ are entire, then there are no singular points, except for the point $p=\infty$.

Remark 20. In a particular case, solutions of (89) are located on the boundary of the stability domain. If $p=0$, then $\Delta(0)=$ 0 is the equation determining the boundary of the stability domain.
Thus, there exist limits

$$
\lim _{t \rightarrow \infty} \int_{0}^{t} \psi_{k}(t-\tau) e^{2 a_{k}(t-\tau)} W_{k}(\tau) d \tau=0, \quad k=1, \ldots, n .
$$

Corollary 19. Let the assumptions of Theorem 18 hold. Then, the asymptotical stability of solutions of system (75) implies the asymptotical mean square stability of the zero solution of (70).

Proof. Under the given assumptions, this follows from the existence of limits (77).

The results obtained make it possible to examine the stability of the stochastic equation (70) by using the deterministic system of (76). Here, we can use the known methods such as the Laplace transformation.

If we denote

$$
\begin{array}{r}
\Upsilon_{k}(p)=\int_{0}^{\infty} e^{-p t} W_{k}(t) d t, \quad \Theta_{k s}(p)=\int_{0}^{\infty} e^{-p t} q_{k s}(t) d t, \\
k, s=1, \ldots, n,
\end{array}
$$

then, multiplying (76) by $e^{-p t}$ and integrating it from 0 to $\infty$ with respect to $t,(76)$ can be transformed into the system of linear algebraic equations with respect to the functions $\Upsilon_{k}(p)$. We get the system

$$
\begin{aligned}
\Upsilon_{k}(p)= & \sum_{s=1}^{n} \Theta_{k s}\left(p-2 a_{s}\right) C_{k s}^{2} E_{s}^{(2)}\{x(0)\} \\
& +\sum_{s=1}^{n} \Theta_{k s}\left(p-2 a_{s}\right) C_{k s}^{2} \Upsilon_{s}(p),
\end{aligned}
$$

where $k=1, \ldots, n$

The determinant $\Delta(p)$ of the system of linear equations (89) is in the form
To solve the model problem formulated at the beginning of this section, we use the stochastic operators $S_{s k} f(x), s, k=$ $1,2, \ldots, n$ in the form

$$
S_{s k} f(t, x)= \begin{cases}f(t, x), & s=k, \\ \sum_{k=1}^{n} \frac{p_{k}}{p_{s}} f\left(t, \frac{x}{p_{s}}\right), & s \neq k,\end{cases}
$$


associated to the intensities $q_{s k}(t)$ determined by (72). The domain of stability of banking operations in the foreign currency exchange market can be derived from the behavior of the solutions of the moment equations (76). Before we use the moment equations, we express the probabilities $C_{k s}$, $k, s=1,2, \ldots, n$ from formula (14) by using the probabilities $p_{k}, k=1,2, \ldots, n$ from formula (74) in the form

$$
C_{k s}= \begin{cases}1, & s=k \\ \sum_{k=1}^{n} p_{k}^{2}, & s \neq k\end{cases}
$$

Then, the moment equations (76) can be rewritten into the form

$$
\begin{aligned}
W_{k}(t)=\rho\left[\sum_{\substack{s=1 \\
s \neq k}}^{n} q_{k s}(t) e^{2 a_{s} t} E_{s}^{(2)}\{x(0)\}\right. & \\
& \left.+\int_{0}^{t} \sum_{s=1}^{n} q_{k s}(t-\tau) e^{2 a_{s}(t-\tau)} W_{s}(\tau) d \tau\right], \\
k=1, \ldots, n, &
\end{aligned}
$$

where $\rho=\sum_{k=1}^{n} p_{k}^{2}$. The system (93) can be solved in the same way as above, that is, by Laplace transformation. Then, multiplying (93) by $e^{-p t}$ and integrating it from 0 to $\infty$ with respect to $t$, we get a preliminary form of the system

$$
\begin{aligned}
& \Upsilon_{k}(p) \\
& =\rho\left[\int_{0}^{\infty} \sum_{\substack{s=1 \\
k \neq s}}^{n} q_{k s} e^{2 a_{s} t} e^{-p t} E_{s}^{(2)}\{x(0)\} d t\right. \\
& \left.+\int_{0}^{\infty} \int_{0}^{t} \sum_{\substack{s=1 \\
k \neq s}}^{n} q_{k s}(t-\tau) e^{2 a_{s}(t-\tau)} e^{-p t} W_{s}(\tau) d \tau d t\right], \\
& k=1, \ldots, n,
\end{aligned}
$$

which we will still have to modify using some properties of the Laplace transformation. In accordance with the property of the delay and with regard to the equality

$$
\int_{0}^{\infty} q_{k s} e^{-p t} d t=\frac{1-e^{-p T_{k s}}}{T_{k s} p}, \quad k, s=1, \ldots, n, k \neq s,
$$

we obtain

$$
\int_{0}^{\infty} q_{k s} e^{2 a_{s} t} e^{-p t} d t=\frac{1-e^{-T_{k s}\left(p-2 a_{s}\right)}}{T_{k s}\left(p-2 a_{s}\right)}, \quad k, s=1, \ldots, n, k \neq s .
$$

In accordance with the property of convolution, we get

$$
\int_{0}^{\infty} \int_{0}^{t} q_{k s}(t-\tau) e^{2 a_{s}(t-\tau)} e^{-p t} W_{s}(\tau) d \tau d t=\frac{1-e^{-T_{k s}\left(p-2 a_{s}\right)}}{T_{k s}\left(p-2 a_{s}\right)} .
$$

Therefore, the system of (94) can be written in the form

$$
\begin{aligned}
\Upsilon_{k}(p)=\rho\left[\sum_{\substack{s=1 \\
k \neq s}}^{n} E_{s}^{(2)}\{x(0)\} \frac{1-e^{-T_{k s}\left(p-2 a_{s}\right)}}{T_{k s}\left(p-2 a_{s}\right)}\right. \\
\left.+\sum_{\substack{s=1 \\
k \neq s}}^{n} f_{s}(p) \frac{1-e^{-T_{k s}\left(p-2 a_{s}\right)}}{T_{k s}\left(p-2 a_{s}\right)}\right],
\end{aligned}
$$

where $k=1, \ldots, n$, or, using the notations

$$
\begin{gathered}
b_{k}(p) \equiv \sum_{\substack{s=1 \\
k \neq s}}^{n} E_{s}^{(2)}\{x(0)\} \frac{1-e^{-T_{k s}\left(p-2 a_{s}\right)}}{T_{k s}\left(p-2 a_{s}\right)}, \\
a_{k s} \equiv \frac{1-e^{-T_{k s}\left(p-2 a_{s}\right)}}{T_{k s}\left(p-2 a_{s}\right)},
\end{gathered}
$$

$k, s=1, \ldots, n, k \neq s$, in a simpler form

$$
\Upsilon_{k}(p)=\rho\left(b_{k}(p)+\sum_{\substack{s=1 \\ k \neq s}}^{n} a_{k s} \Upsilon_{s}(p)\right), \quad k=1, \ldots, n .
$$

By the Cramer theorem, we can solve the system (100). The singular points are determined by the roots of

$$
\operatorname{det}(E-\rho a)=0 \text {, }
$$

where $a \equiv\left(a_{k s}\left(p, T_{k s}\right)\right)_{k, s=1}^{n}$, while $\rho a_{k k}\left(p, T_{k k}\right) \equiv-1$.

The character of the roots of (101) determines the stability of the solutions of the system of integral equations in (93). If the real parts of all the roots of (101) are negative, then the solutions of (93) are asymptotically stable. If there is at least one root of (101) with a positive real part, then the solutions of integral equations (93) are unstable.

The character of the dependence between parameters $p$ and $T_{k s}$ can be determined by solving the system of algebraic equations in (101) by numerical methods.

Example 21. The real boundaries of the instability domain of foreign currency exchange market can be determined in a particular case. Suppose that the random semi-Markov process can take three states:

$\theta_{1}$-if the bank operates in a currency crisis, then $a(\xi(t))=a_{1}$;

$\theta_{2}$-if the bank operates in a stable foreign currency exchange market, then $a(\xi(t))=a_{2}$;

$\theta_{3}$-if the bank operates in a market with currency restrictions, then $a(\xi(t))=a_{3}$,

with intensities

$$
\begin{gathered}
q_{11}(t)=q_{22}(t)=q_{33}(t) \equiv 0, \\
q_{12}(t)=q_{13}(t)=q_{21}(t)=q_{23}(t)=q_{31}(t) \\
=q_{32}(t) \equiv \begin{cases}\frac{1}{T} & \text { for } 0 \leq t<T, \\
0 & \text { for } t>T,\end{cases}
\end{gathered}
$$




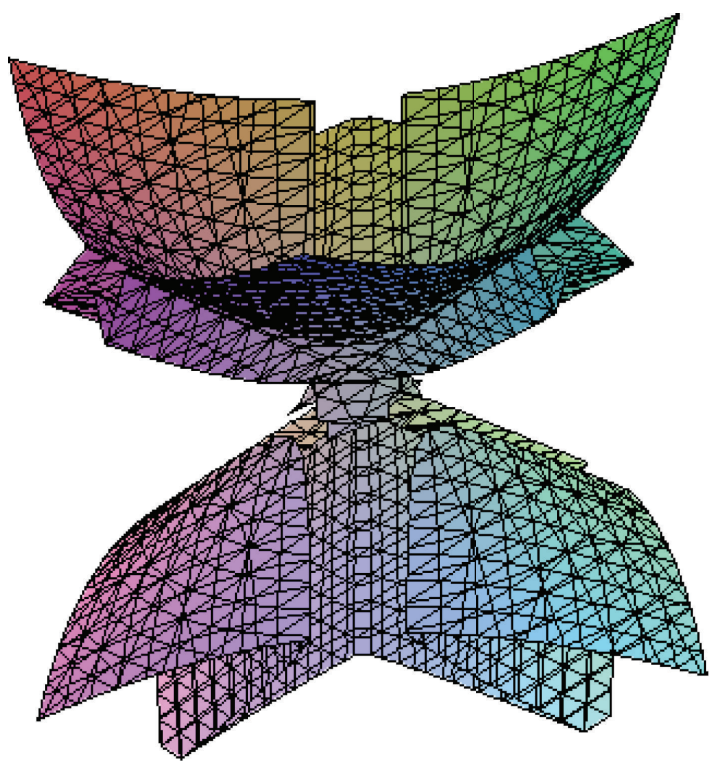

(a)

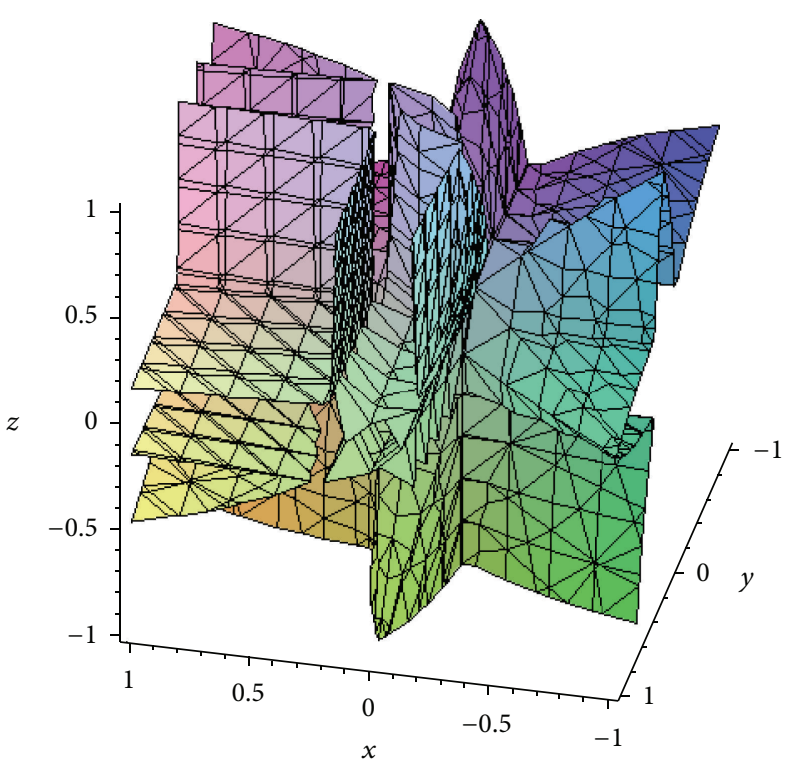

(b)

FIGURE 1: The boundary of instability of solutions of (70) constructed in the plane of parameters $a_{1}, a_{2}$, and $a_{3}$ for $p=0$ and for different values of $\rho$.

which means that the bank remains in each state for the same period of time $1 / T$. In the above case, the system (100) takes the form

$$
\begin{aligned}
& \Upsilon_{1}(p)=\rho b_{1}(p)+\rho a_{12} \Upsilon_{2}(p)+\rho a_{13} \Upsilon_{3}(p), \\
& \Upsilon_{2}(p)=\rho b_{2}(p)+\rho a_{21} \Upsilon_{1}(p)+\rho a_{23} \Upsilon_{3}(p), \\
& \Upsilon_{3}(p)=\rho b_{3}(p)+\rho a_{31} \Upsilon_{1}(p)+\rho a_{32} \Upsilon_{2}(p),
\end{aligned}
$$

where

$$
\begin{array}{ll}
a_{12}=\frac{1-e^{-T\left(p-2 a_{2}\right)}}{T\left(p-2 a_{2}\right)}, & a_{13}=\frac{1-e^{-T\left(p-2 a_{3}\right)}}{T\left(p-2 a_{3}\right)}, \\
a_{21}=\frac{1-e^{-T\left(p-2 a_{1}\right)}}{T\left(p-2 a_{1}\right)}, & a_{23}=\frac{1-e^{-T\left(p-2 a_{3}\right)}}{T\left(p-2 a_{3}\right)}, \\
a_{31}=\frac{1-e^{-T\left(p-2 a_{1}\right)}}{T\left(p-2 a_{1}\right)}, & a_{32}=\frac{1-e^{-T\left(p-2 a_{2}\right)}}{T\left(p-2 a_{2}\right)} .
\end{array}
$$

The value $\rho$ expresses the mean value of the bank's income from foreign currency transactions during time $T$. The singular point is $p=0$, when the solution is situated on the boundary of the domain of instability on the plane of coefficients $a_{1}, a_{2}$, and $a_{3}$.

Equation (101) is in the form

$$
\left|\begin{array}{ccc}
1 & -\rho a_{12} & -\rho a_{13} \\
-\rho a_{21} & 1 & -\rho a_{23} \\
-\rho a_{31} & -\rho a_{32} & 1
\end{array}\right|=0
$$

or

$$
\begin{gathered}
1-\rho^{3} a_{21} a_{32} a_{13}-\rho^{3} a_{12} a_{23} a_{31}-\rho^{2} a_{13} a_{31} \\
-\rho^{2} a_{23} a_{32}-\rho^{2} a_{12} a_{21}=0 .
\end{gathered}
$$

If $p=0$, the boundaries of instability of solutions of (70) are constructed in the plane of parameters $a_{1}, a_{2}$, and $a_{3}$ for different values $\rho$ (see Figure 1 where some admissible boundaries are constructed).

\section{Acknowledgment}

The first author was supported by the Grant no P201/11/0768 of Czech Grant Agency (Prague).

\section{References}

[1] F. E. Benth, V. A. Kholodnyi, and P. Laurence, Eds., Quantative Energy Finance: Modelling, Pricing and Heading in Energy, Springer, 2013.

[2] H. Dong and X. Zhao, "Numerical method for a Markovmodulated risk model with two-sided jumps," Abstract and Applied Analysis, vol. 2012, Article ID 401562, 9 pages, 2012.

[3] C. Huang, C. Peng, X. Chen, and F. Wen, "Dynamics analysis of a class of delayed economic model," Abstract and Applied Analysis, vol. 2013, Article ID 962738, 12 pages, 2013.

[4] J. Diblík, I. A. Dzhalladova, M. Michalková, and M. Růžičková, "Modeling of applied problems by stochastic systems and their analysis using the moment equations," Advances in Difference Equations, vol. 2013, article 152, 2013.

[5] I. A. Dzhalladova, "Estimates of stability of functional equation of neutral type wih random right part," TWMS Journal of Pure and Applied Mathematics, vol. 4, pp. 61-68, 2013.

[6] I. Dzhalladova, M. Michalková, and M. Růžičková, "Model of stabilizing of the interest rate on deposits banking system using by moment equations," Tatra Mountains Mathematical Publications, vol. 54, pp. 45-59, 2013. 
[7] T. Biswas, "Stochastic dominance and comparative risk aversion," Review of Economic Analysis, vol. 4, pp. 105-122, 2012.

[8] T. Watanabe, "Net worth ratio and financial instability," Journal of Economic Structures, vol. 2, article 3, 2013. 


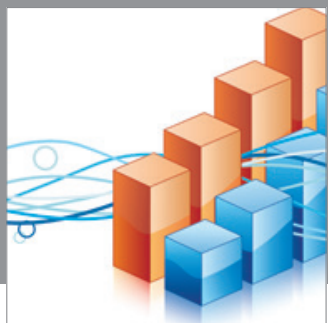

Advances in

Operations Research

mansans

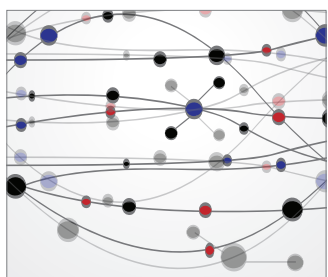

The Scientific World Journal
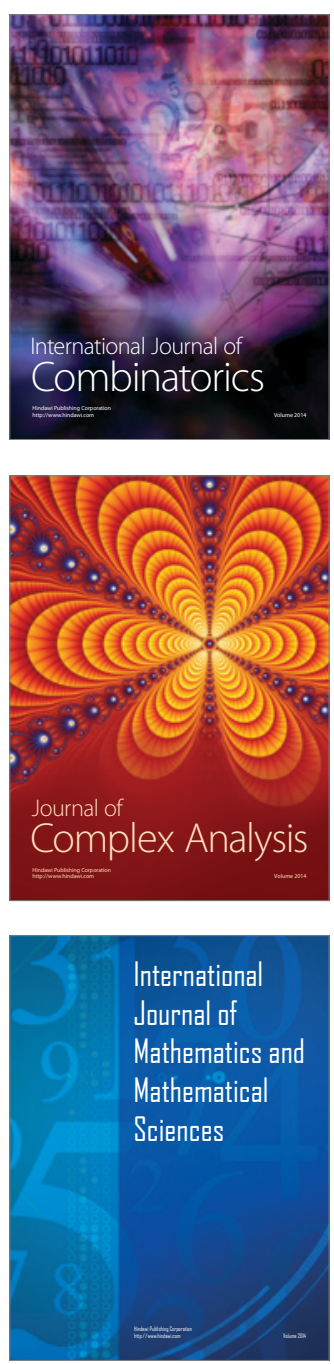
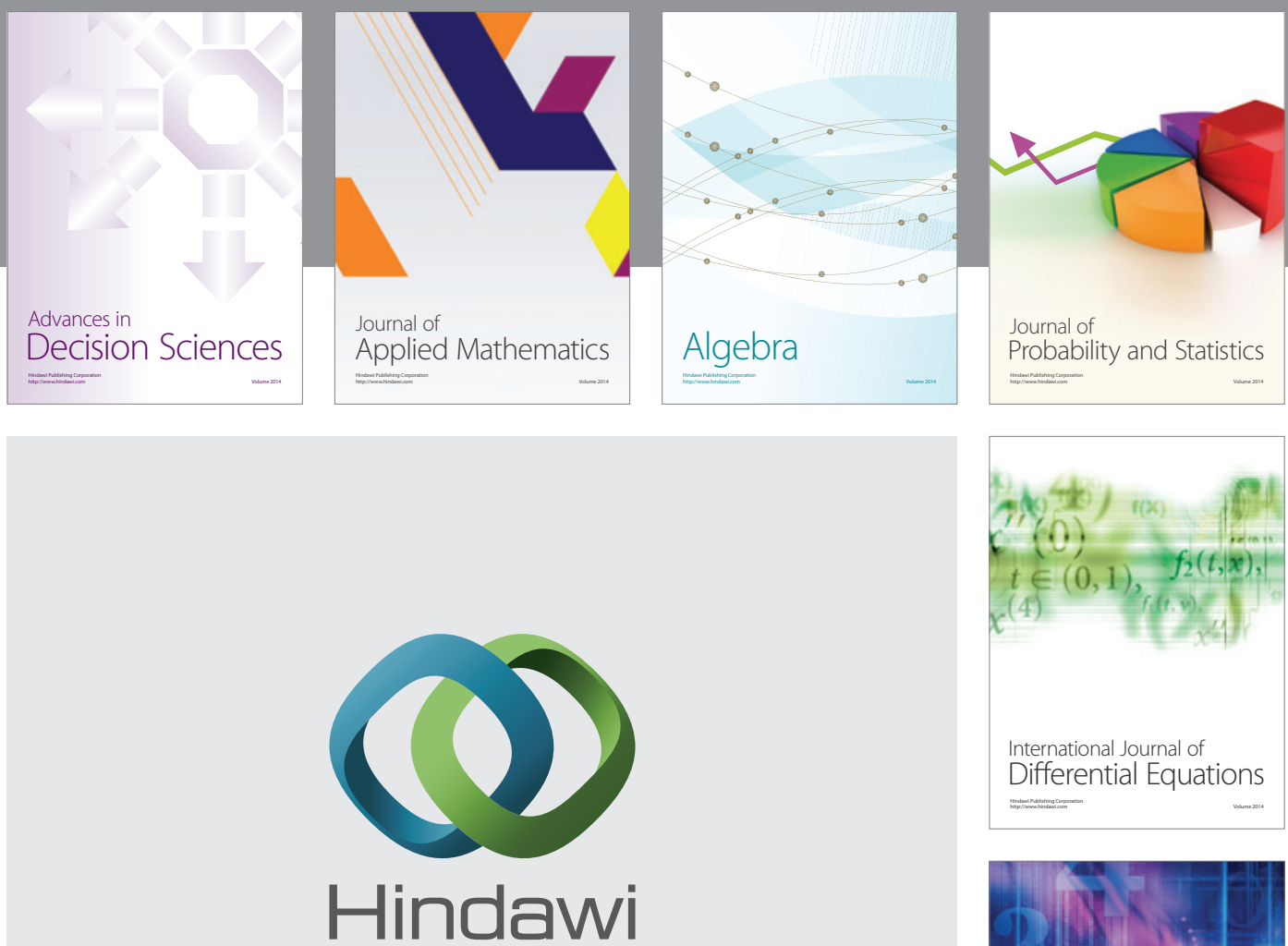

Submit your manuscripts at http://www.hindawi.com
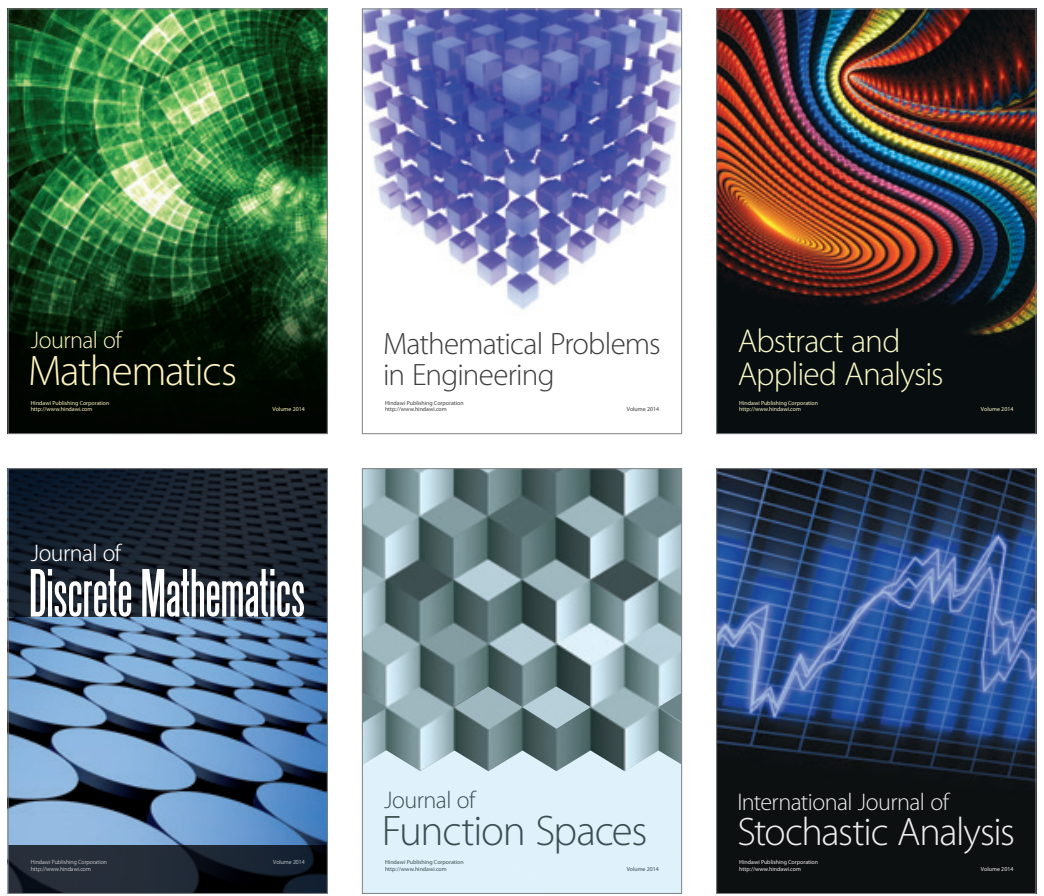

Journal of

Function Spaces

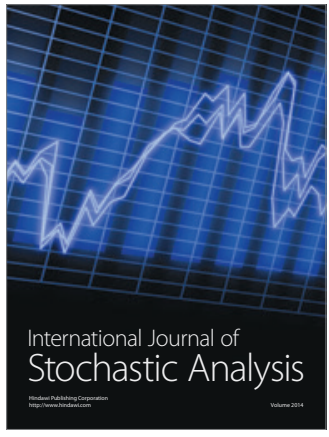

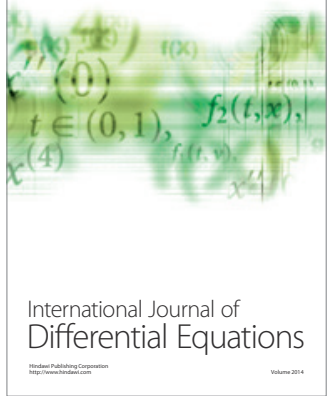
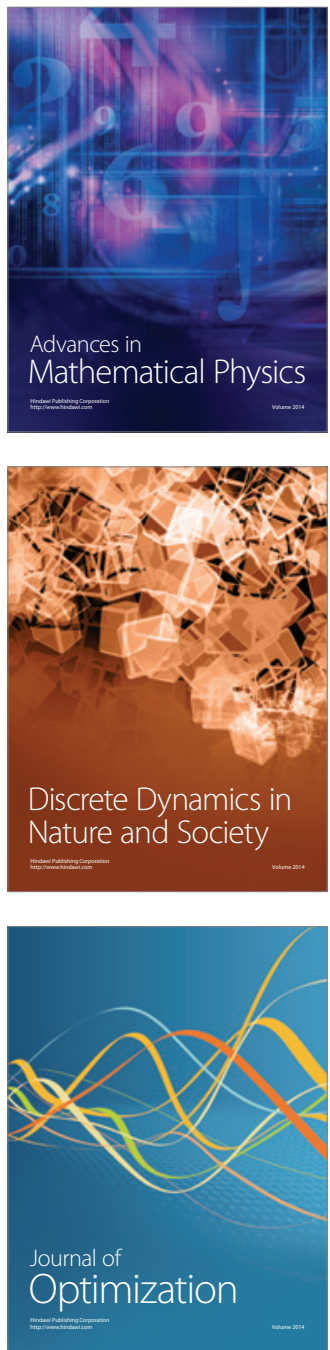